\title{
A Hint to Possible Anisotropy in Radio Universe
}

\author{
Rendong Nan and Zhengdong Cai * \\ Beijing Astronomical Observatory, Beijing-100080, P.R. China
}

\begin{abstract}
This paper presents our investigations of statistical behavior of the small-scale jet orientations and the intrinsic polarization position angles. We found that the distribution of the milliarcsecond-scale jet orientations and intrinsic polarization position angles of extragalatic radio sources appears anisotropic with high significance levels in most cases.
\end{abstract}

Key words: Radio sources: Isotropy - Jet - Polarization position angles

\section{Projected Orientation of Radio Jets in the sky}

We started with an analysis of the data which came from the results of a long-term study by Aller et al. (1992) on the sources in the Pearson-Readhead VLBI survey (1981). The distribution of jet position angles on small scale determined by VLBI is summaried in figure 1. in which, two-thirds of the sample sources ( 26 of 39 sources) have the righthanded structures, and only 3 out of 39 sources are within the second quadrant. We apply binomial and $\chi^{2}$ tests to the righthanded event and the unusual sparseness of jet orientations in the second quadrant, which yield significance levels $\alpha=1.3 \times 10^{-2}$ and 0.1 respectively. The similar results are also found in the Polatidies' 1 Jy sample (1993) for which measurements of the small-scale position angles are available. observations. Probably a description of the anisotropic orientation of jets, in more precise terms, should be revised as that the radio jets in the survey seem to avoid the second quadrant.

The relation between bright cores and relativistic beaming prompts us to check the jet orientation in those superluminals. There are 13 superluminal sources in the samples, among which, 9 out of 13 are righthanded. None is found in the second quadrant. We have also made statistics of all superluminal sources whose orientation information is available to us. Figure 2 presents the orientations of radio jets of the superluminal sources. 22 out of 36 sources are righthanded and only 2 are located in second quadrant, suggesting rather identical feature compared to figure 1 .

\section{Polarization Position Angles}

The relations between polarization direction and structures of different types of sources on mas-scale also turn to exist (Gabuzda et al. 1989). For example, the magnetic fields in the mas-scale jets are perpendicular to the jet orientations in BL Lacs and parallel in radio quasars. Considering the geometrical relation between radio jets and magnetic fields, we examined the distribution

\footnotetext{
* The project is supported by the National Natural Science Foundation of China.
}

491

M. Kafatos and Y. Kondo (eds.), Examining the Big Bang and Diffuse Background Radiations, 491-492. (c) 1996 IAU. Printed in the Netherlands. 
of polarization position angles to take the possible chance in verifying the isotropy of jet orientation. The data from the Aller's paper are graphically presented in figure 3. The distribution of the polarization position angles corrected for Faraday rotation is dramatically peaked at around $90^{\circ}$ and clusters the angles of about 80 percent of sources within 60 degrees from $60^{\circ}$ to $120^{\circ}$. The $\chi^{2}$ test of figure 3 for eight degrees of freedom yielding a significance level of $5.0 \times 10^{-3}$. We extended the studies to a larger sample of 555 radio sources (Simard-Normandin et al. 1981). In figure 4, the intrinsic polarization position angles obviously tend to prefered the horizontal and vertical directions with the significance levels of 0.045 and 0.38 respectively.

The results are primitive. Elaborative inspection of large sample is definitely required for a more convincing conclusion. The main purpose of this work is to draw attention to those careless omissions in observations, and to invite the verification of the imperfections in method and technique which may cause the irregularity in spite of the implausibility. Although the reality of the phenomena is hardly thinkable, "the universe is not only stranger than we know, it is stranger than what we can know".

\section{References}

Aller, M.F., Aller, H.D., and Hughes, P.A., 1992, Ap.J., 399, 16

Esko Valtaoja and Mauri Valtonen, 1992, Variability of Blazars, Combridge Univ. Press. Gabuzda, D.C., Cawthorne, T.V., Roberts, D.H., and Wardle, J.F.C., 1992, Ap.J., 388, 40 Polatidis, A.G., 1993, Ph.D thesis, Univ. of Manchester

Simard-Normandin,M., Kronberg, P.P., and Button, S., 1981, Ap.J.Supple., 45, 97

Zensus J.A. and Pearson T.J., 1987, Superluminal Radio Sources, Cambridge Univ. Press.
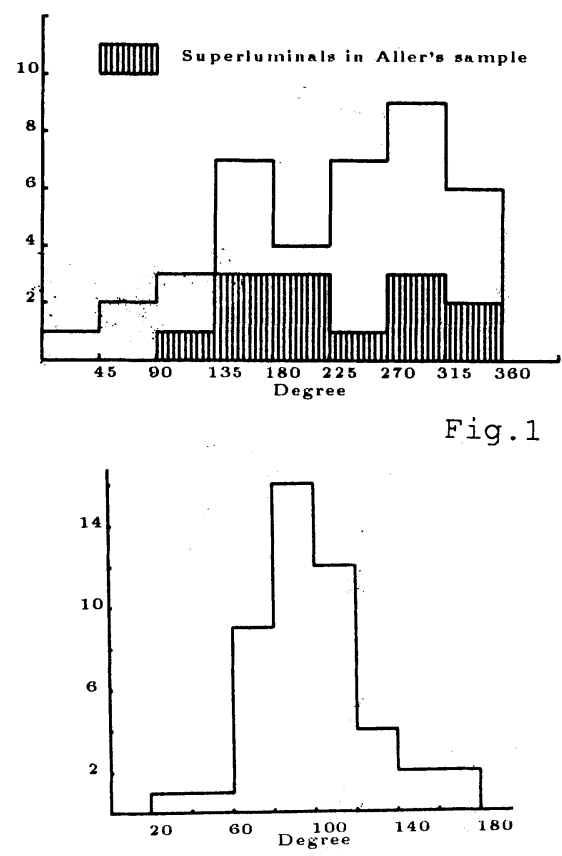

Fig. 3

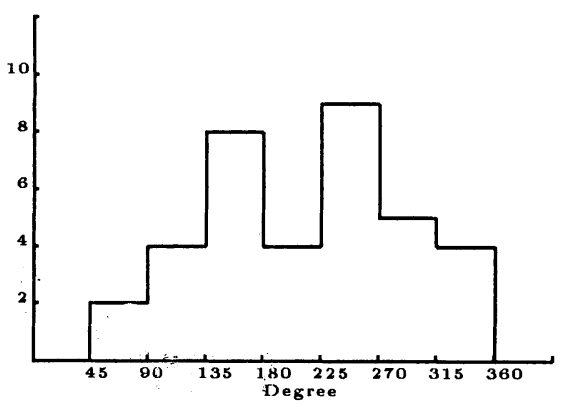

Fig.. 2

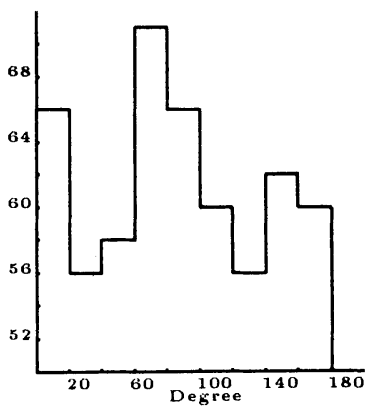

Fig. 4 